\title{
Case Report \\ Pulmonary Edema in Myasthenic Crisis
}

\author{
Uttara Swati Anand, ${ }^{1}$ Stalin Viswanathan, ${ }^{2}$ and Jayanthi Arulneyam ${ }^{3}$ \\ ${ }^{1}$ Department of Internal Medicine, Pondicherry Institute of Medical Sciences, Kalapet, Pondicherry 605009, India \\ ${ }^{2}$ Department of General Medicine, Indira Gandhi Medical College, Kathirkamam, Pondicherry 605009, India \\ ${ }^{3}$ Department of Neurology, Pondicherry Institute of Medical Sciences, Kalapet, Pondicherry 605009, India
}

Correspondence should be addressed to Stalin Viswanathan; stalinviswanathan@ymail.com

Received 14 September 2013; Accepted 9 October 2013

Academic Editors: C. Diez, C. D. Roosens, and J. Starkopf

Copyright (C) 2013 Uttara Swati Anand et al. This is an open access article distributed under the Creative Commons Attribution License, which permits unrestricted use, distribution, and reproduction in any medium, provided the original work is properly cited.

We report a previously asymptomatic 50-year-old lady who came with myasthenic crisis as initial presentation of myasthenia gravis. She developed pulmonary edema following intravenous immunoglobulin administration and had ischemic changes in ECG and left ventricular dysfunction on echocardiography. She improved with diuretics, dobutamine, and fluid restriction alone. This is the first report in English-language medical literature describing the association between myasthenic crisis and likely takotsubo cardiomyopathy-related pulmonary edema following intravenous immunoglobulin administration.

\section{Introduction}

Myasthenia gravis (MG) is an antibody-mediated neurological disorder that affects the neuromuscular junction [1]. Myasthenic crisis is characterized by respiratory failure that requires mechanical ventilation [2]. Takotsubo cardiomyopathy is defined as a profound but reversible left ventricular dysfunction in the absence of coronary artery disease [3]. We present a 50-year-old lady with myasthenic crisis (MC), who developed pulmonary oedema following intravenous immunoglobulin (IVIG) and was found to have features suggestive of takotsubo cardiomyopathy (TC).

\section{Case}

She had had cough, rhinorrhoea, headache, myalgias, and fever for 10 days and presented to another hospital with fatigue of a week's duration, breathlessness, slurring of speech, and nasal regurgitation of liquids for two days. She was intubated and then transferred to our institution. On admission, she was conscious and afebrile with tachycardia $(110 / \mathrm{min})$, tachypnoea $(22 / \mathrm{min})$, and normal blood pressure. Cardiovascular and respiratory system examination was otherwise normal. Neurologically, she had flaccid quadriparesis (2/5), floppy neck, and bilateral facial nerve paralysis without ocular weakness or bladder incontinence.
Chest radiograph (CXR), electrocardiogram (ECG), renal and liver function tests, and haemogram were normal. An initial diagnosis of Guillain-Barre syndrome was made and intravenous immunoglobulin (IVIG) was initiated at a dose of $0.4 \mathrm{~g} / \mathrm{kg} /$ day. On the second day she had asymmetrical ptosis; $1 \mathrm{mg}$ neostigmine administration led to improvement in ptosis and muscle power (4-/5) and neostigmine $30 \mathrm{mg}$ every 4 th hour was continued thereafter. Later during the day, tachycardia, tachypnoea, hypotension $(96 / 60 \mathrm{mmHg})$, and left-sided infra-axillary crackles were observed. Chest radiograph revealed infiltrates in the left mid-zones (Figure 1(a)); ECG showed occasional VPCs (Figure 2(a)), and her total leukocyte counts were $20 \times 10^{9} / \mathrm{L}$. Respiratory alkalosis was seen on arterial blood gas $(A B G)$ analysis. Ceftriaxone, metronidazole, and levofloxacin were administered with a diagnosis of probable aspiration pneumonia; intravenous fluids were infused to maintain CVP of $\sim 10 \mathrm{~cm}$ saline. Eight hours later her endotracheal tube was filled with frothy sputum and her tachypnea worsened. A repeat CXR revealed bilateral hilar infiltrates (Figure 1(b)), while ST depression and $\mathrm{T}$-wave inversion were observed in the lateral leads (Figure 2(b)). IVIG was discontinued on day 3 due to financial constraints. Echocardiography showed hypokinetic anterior wall, distal septum and apex with an ejection fraction of $35 \%$; creatine kinase MB (CK-MB) was $21 \mathrm{U}$ and Trop-I was negative. Her relatives did not provide consent to subject 


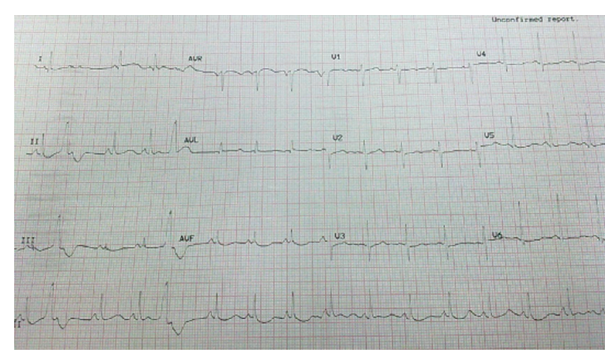

(a)

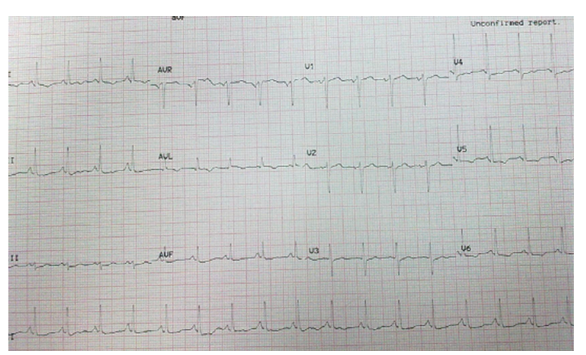

(b)

FIGURE 1: (a) ECG showing VPCs. (b) ECG eight hours later with T-wave inversion and ST depression in leads I, aVL, $\mathrm{V}_{5}$, and $\mathrm{V}_{6}$.

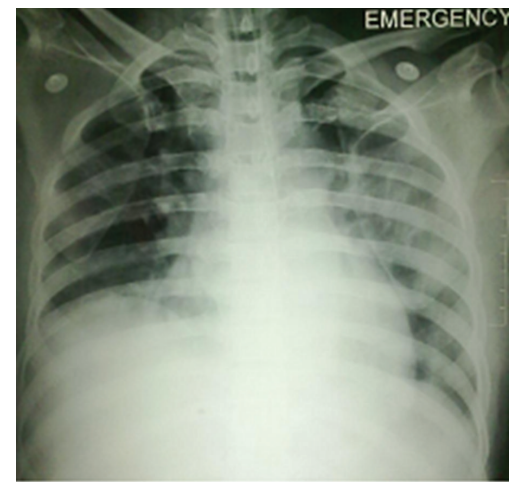

(a)

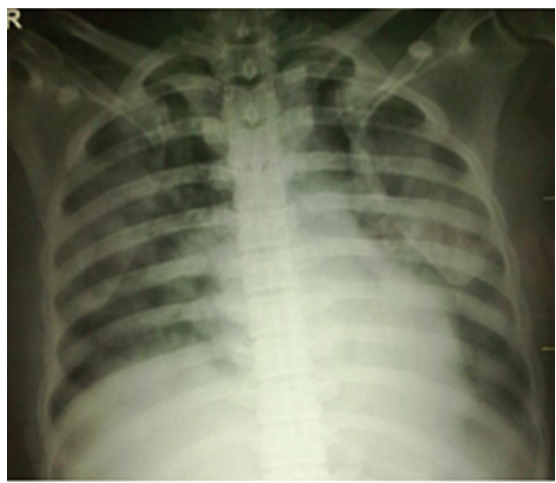

(b)

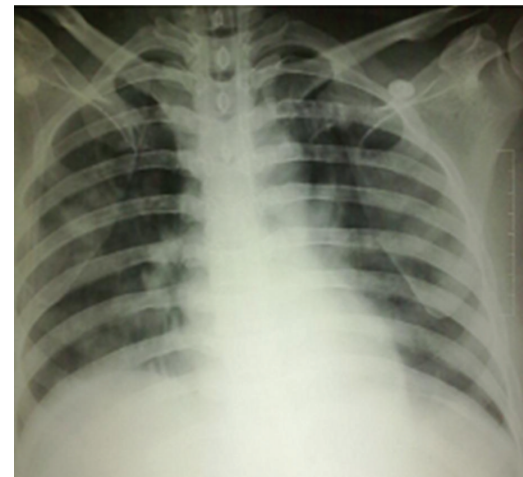

(c)

FIgURE 2: (a) CXR revealing left hilar infiltrates. (b) CXR eight hours later with bilateral hilar infiltrates. (c) CXR 24 hours later with reduction in bilateral hilar infiltrates following diuretics and dobutamine.

the patient for a coronary angiography; stress thallium scintigraphy was unavailable in our hospital. She improved thereafter with dobutamine, diuretics, and fluid restriction following which her CXR showed improvement. An electromyogram and repetitive nerve stimulation performed on day 4 confirmed the diagnosis of myasthenia gravis. ECG normalized on the fifth day. She was found to be ANA and dsDNA positive, while the thyroid profile was normal. Twentyfour-hour urinary protein was $235 \mathrm{mg}$. She did not satisfy any other ACR criteria for SLE. Contrast CT for thymoma was noncontributory. We did not have facility for testing autoantibodies for MG. She was extubated 10 days after admission and was discharged home on oral prednisolone and neostigmine. Echocardiography prior to her discharge revealed an ejection fraction of $55 \%$ with no regional wall motion abnormalities.

\section{Discussion}

A dysfunctional left ventricle resembles a "takotsubo," a narrow-necked pot used to trap octopuses. There are ten reports (between 2005 and 2013) detailing the association of MG and TC, with only five in English medical literature [3]. Three reports from Japan are available-the first following plasmapheresis to treat $\mathrm{MC}$, the second after disopyramide therapy to treat atrial fibrillation (reported in English literature), and the last that developed after thymectomy [3, 4]. The Spanish patient presented with respiratory failure and takotsubo cardiomyopathy and was subsequently diagnosed with myasthenic crisis, while the patient from Brazil was a known case of MG and developed crisis that was treated with plasmapheresis $[5,6]$. Among the cases reported in English literature, none of them had prior therapy with intravenous immunoglobulins $[2,4,7,8]$. Two of them had simultaneous onset of takotsubo cardiomyopathy and myasthenic crisis $[2,9]$.

Our patient presented with myasthenic crisis as initial presentation of MG. She had no ocular symptoms or signs initially, leading us to suspect a different diagnosis. She developed asymmetric pulmonary edema that was treated as aspiration pneumonia initially. IVIG was discontinued after three days due to financial constraints. IVIG can itself cause myocardial infarction and cerebrovascular events as described in some reports. But some of those patients also had risk factors for coronary artery disease. IVIG in myasthenia crisis had caused non-ST elevation myocardial infarction [10]. That patient did not undergo catheterization and had no coronary risk factors; her thallium scan and CT revealed no coronary lesions [5]. IVIG can cause hyperviscosity and is hypothesized to cause coronary vasospasm due to release of cytokines and can cause elevations in catecholamines [10]. Neostigmine in another report was described to precipitate noncardiogenic pulmonary edema neostigmine in our patient was continued even after pulmonary edema without further worsening [11]. Another feature of our patient was the coexistence of SLE autoantibodies and proteinuria with MG. 
Our patient had clinical features of probable TC by virtue of being an older female, with physical and emotional stress of paralysis, hospitalization and mechanical ventilation, IVIG therapy, severe cardiorespiratory symptoms, and signs proportional to echocardiography but dissociated with the severity of ECG changes and cardiac enzymes which would be unlikely in ischemic heart disease. ECG normalized in 48 hours and echocardiography within 10 days. Possibly, IVIG may have a role in inducing TC due to its effects on the heart as seen in other cases although lack of angiography is a severe limitation in our case study. The pulmonary edema may be partly contributed by a neurogenic cause-myasthenia gravis itself. Thus, our patient had probable TC (due to lack of angiographic evidence) following MG and a combination of factors especially IVIG and critical care.

\section{Conclusion}

We report the first patient of MC with probable TC precipitated by immunoglobulins in English-language medical literature. Patients with MG are at potential risk of developing TC due to various physical and emotional stresses that they are subjected to during the course of their treatment. This risk is compounded by the fact that IVIG could also lead to TC by its effects on the heart. Hence, careful clinical and electrocardiographic monitoring is essential in all MG patients on IVIG therapy and coronary artery evaluation may be required prior to IVIG in such patients.

\section{Conflict of Interests}

The authors declare that they have no conflict of interests.

\section{References}

[1] R. Gold and C. Schneider-Gold, "Current and future standards in treatment of myasthenia gravis," Neurotherapeutics, vol. 5, no. 4, pp. 535-541, 2008.

[2] S. R. Beydoun, J. Wang, R. L. Levine, and A. Farvid, "Emotional stress as a trigger of myasthenic crisis and concomitant takotsubo cardiomyopathy: a case report," Journal of Medical Case Reports, vol. 4, article 393, 2010.

[3] J. Finsterer and C. Stöllberger, "Neuromuscular disorders and Takotsubo syndrome," International Journal of Cardiology, vol. 168, no. 4, pp. 4293-4294, 2013.

[4] K. Hirose, H. Yamaguchi, Y. Oshima et al., "Severe respiratory failure and torsades de pointes induced by disopyramide in a patient with myasthenia gravis," Internal Medicine, vol. 47, no. 19, pp. 1703-1708, 2008.

[5] S. Mayor-Gomez, F. Lacruz, and D. Ezpeleta, "Myasthenic crisis and Takotsubo syndrome: a non-chance relationship," Revue Neurologique, vol. 55, pp. 725-728, 2012.

[6] J. M. A. de Sousa, M. Knobel, G. Buchelle et al., "Transient ventricular dysfunction (Takotsubo cardiomyopathy)," Arquivos Brasileiros de Cardiologia, vol. 84, no. 4, pp. 340-342, 2005.

[7] C. P. Wong and P. L. Chia, "Recurrent takotsubo cardiomyopathy precipitated by myasthenic crisis," International Journal of Cardiology, vol. 155, no. 1, pp. ell-e12, 2012.

[8] S. Bijulal, S. Harikrishnan, N. Namboodiri, V. K. Ajitkumar, D. Gupta, and P. S. Mathuranath, "Tako-tsubo cardiomyopathy in a patient with myasthenia gravis crisis: a rare clinical association," BMJ Case Reports, 2009.

[9] V. Bansal, M. M. Kansal, and J. Rowin, "Broken heart syndrome in myasthenia gravis," Muscle and Nerve, vol. 44, no. 6, pp. 990993, 2011.

[10] M. Mizrahi, T. Adar, E. Orenbuch-Harroch, and Y. Elitzur, "Non-ST elevation myocardial infraction after high dose intravenous immunoglobulin infusion," Case Reports in Medicine, vol. 2009, Article ID 861370, 4 pages, 2009.

[11] L. K. Raiger, U. Naithani, B. S. Vijay, P. Gupta, and V. Bhargava, "Non-cardiogenic pulmonary oedema after neostigmine given for reversal: a report of two cases," Indian Journal of Anaesthesia, vol. 54, no. 4, pp. 338-341, 2010. 


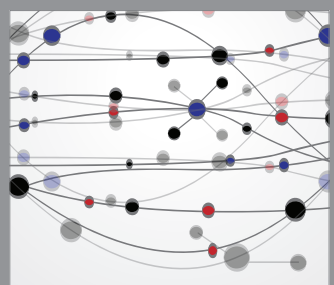

The Scientific World Journal
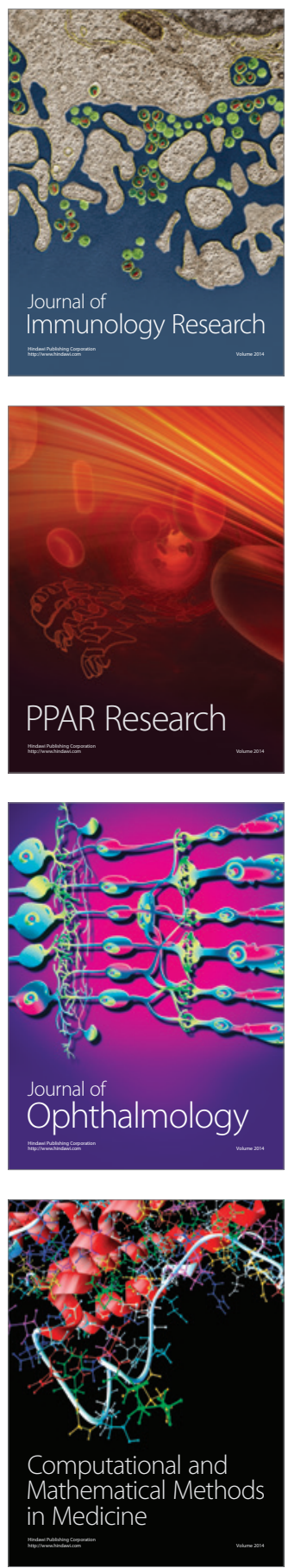

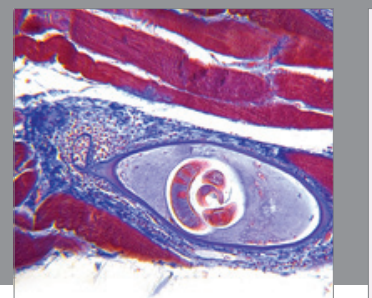

Gastroenterology

Research and Practice
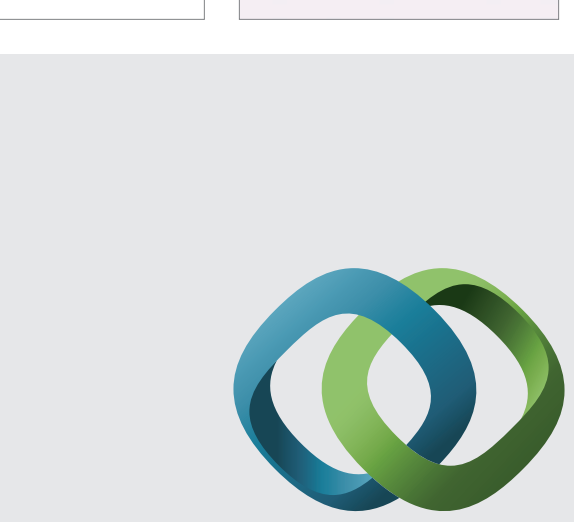

\section{Hindawi}

Submit your manuscripts at

http://www.hindawi.com
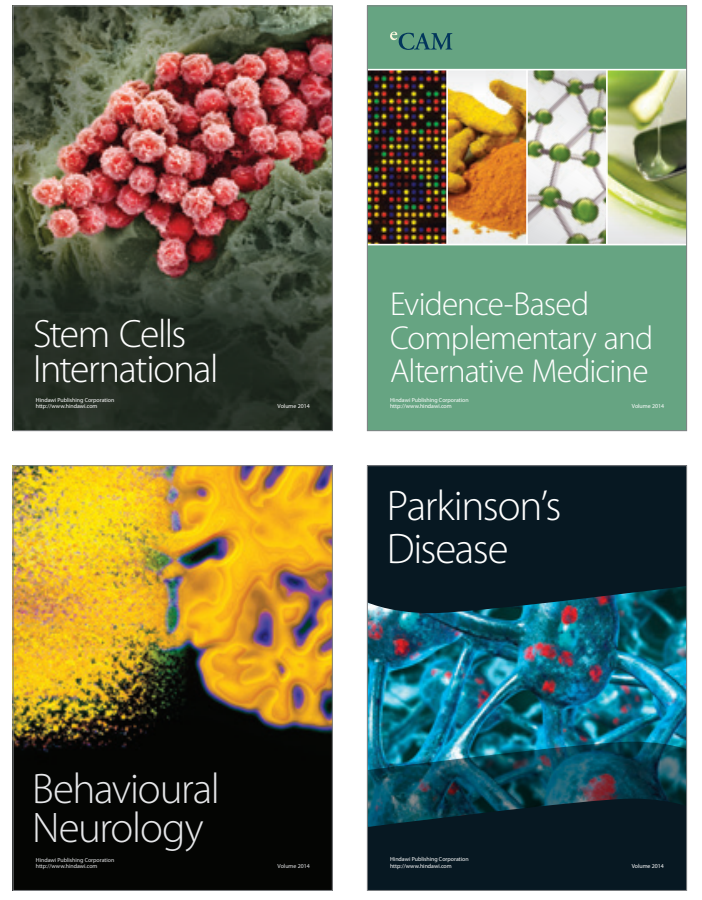
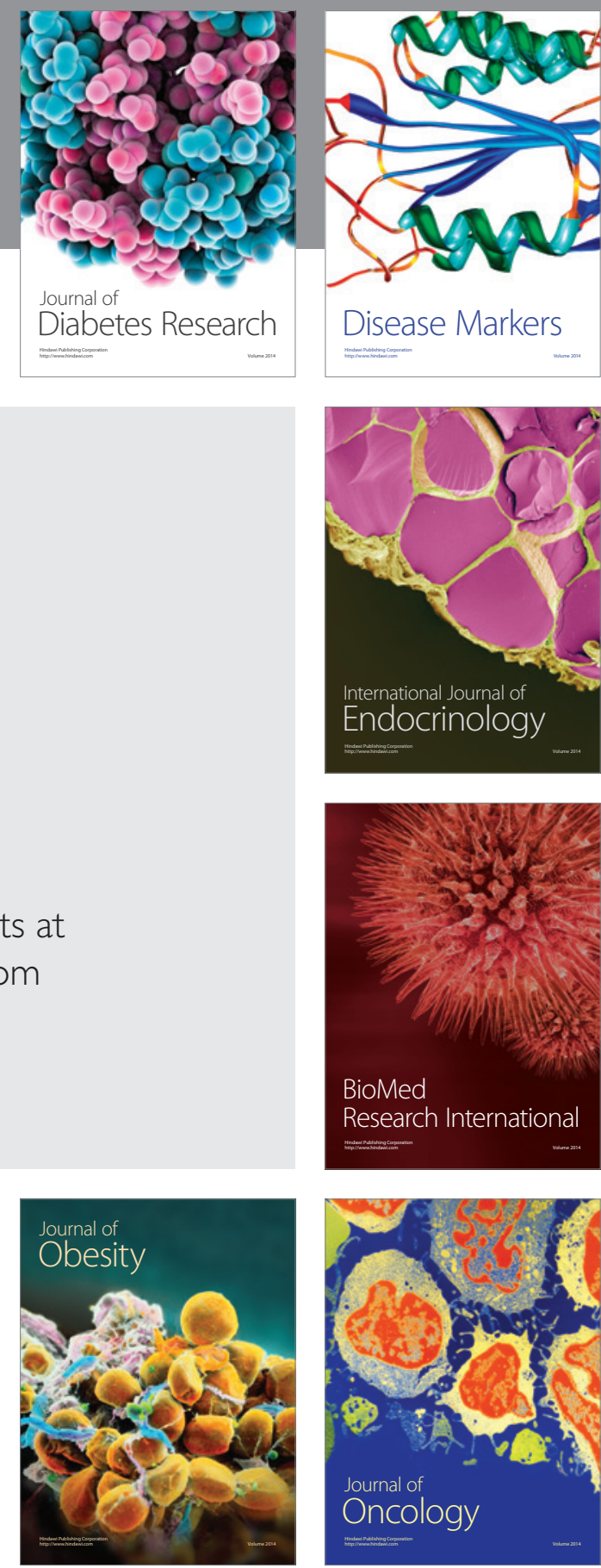

Disease Markers
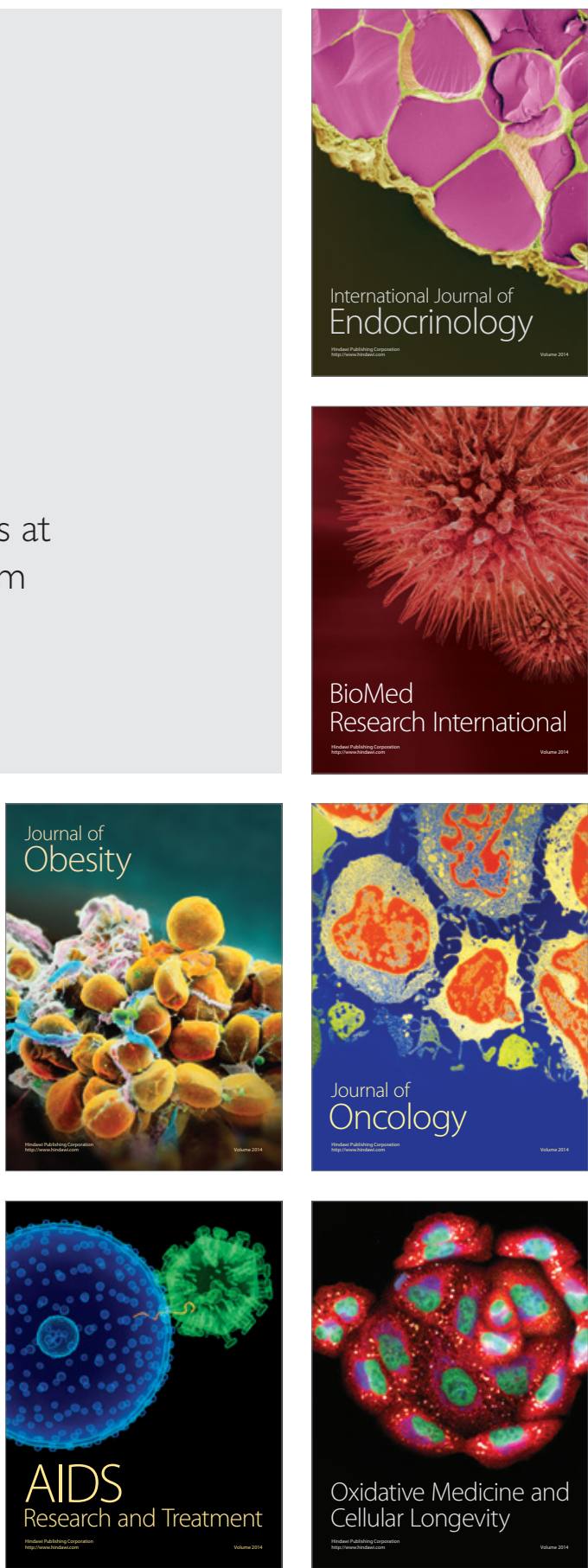